KYUNGPOOK Math. J. 54(2014), 639-653

http://dx.doi.org/10.5666/KMJ.2014.54.4.639

\title{
Finite Type Invariants and the Kauffman Bracket Polynomi- als of Virtual Knots
}

\author{
MYEONG-JU JEONG \\ Department of Mathematics and Computer Science, Korea Science Academy of \\ KAIST, 111 Baekyang Gwanmun-Ro, Busanjin-Gu, Busan 614-822, Korea \\ e-mail : mjjeong@kaist.ac.kr \\ Chan-Young PARK* \\ Department of Mathematics, Kyungpook National University, Taegu, 702-701, Ko- \\ rea \\ e-mail : chnypark@knu.ac.kr \\ SOON TAE YEO \\ Department of Mathematics, Busan National University, Busan, 609-735, Korea \\ e-mail : ysoon6@yahoo.co.kr
}

Abstract. In [9], Kauffman introduced virtual knot theory and generalized many classical knot invariants to virtual ones. For example, he extended the Jones polynomials $V_{K}(t)$ of classical links to the $f$-polynomials $f_{K}(A)$ of virtual links by using bracket polynomials. In [4], M. Goussarov, M. Polyak and O. Viro introduced finite type invariants of virtual knots. In this paper, we give a necessary condition for a virtual knot invariant to be of finite type by using $t\left(a_{1}, \cdots, a_{m}\right)$-sequences of virtual knots. Then we show that the higher derivatives $f_{K}^{(n)}(a)$ of the $f$-polynomial $f_{K}(A)$ of a virtual knot $K$ at any point $a$ are not of finite type unless $n \leq 1$ and $a=1$.

\section{Introduction}

L. H. Kauffman introduced virtual knots, a generalization of knots motivated from knots in thickened surfaces and abstract properties of Gauss code, by considering virtual crossings of knot diagrams and introduced graphical finite type invariants of virtual knots ([9]). In 2000, M. Goussarov, M. Polyak and O. Viro introduced a different kind of finite type invariants of virtual knots by using semi-virtual cross-

* Corresponding Author.

Received August 20, 2013; accepted December 31, 2013.

2010 Mathematics Subject Classification: 57M25, 57M27.

Key words and phrases: virtual knots, graphical finite type invariants, finite type invariants of virtual knots. 
ings instead of singular points in Vassiliev invariants theory ([4]). For a given knot diagram, we can characterize it by using a diagram of a circle with some signed arrows as following. For a diagram of a knot $K$, consider a diagram of a circle connected by chords whose endpoints are the double points of the projection of the knot diagram. Each chord in the Gauss diagram is oriented from the upper strand to the lower strand in the crossing and it is also equipped with the sign of the corresponding crossing. We call the diagram of the circle the Gauss diagram of the knot $K$. We consider a Gauss diagram up to orientation preserving homeomorphism of the embedding of the underlying circle. See Figure 1.

Throughout this paper we denote the sets of all nonnegative integers, integers and real numbers by $\mathbb{N}, \mathbb{Z}$ and $\mathbb{R}$ respectively.
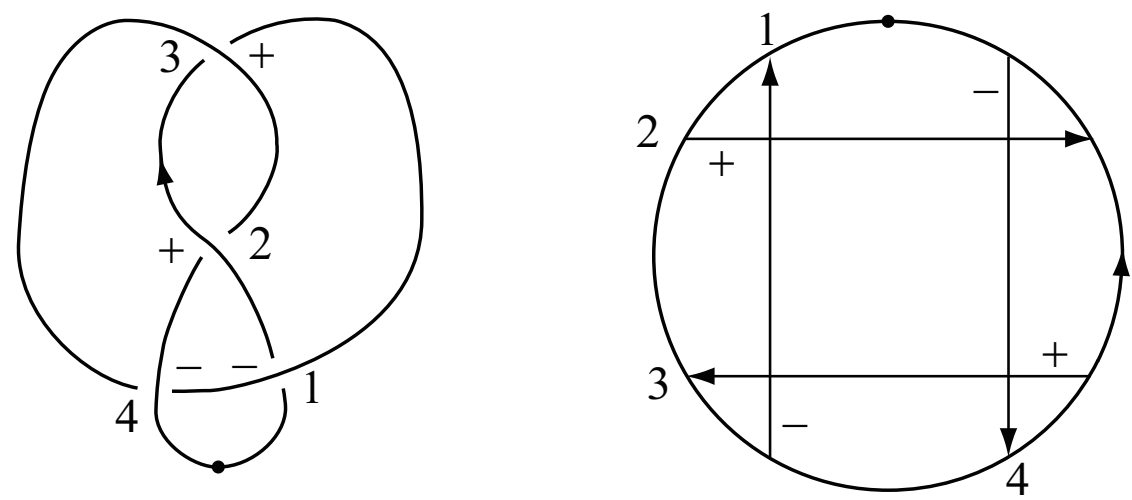

Figure 1

A virtual knot diagram is a knot diagram with classical crossings and virtual crossings. We denote a virtual crossing by a singular point surrounded by a small circle as shown in Figure 2. For a virtual knot diagram $K$, we define its Gauss diagram $G(K)$ to be the diagram with signed and oriented chords following the construction of a Gauss diagram for a classical one, disregarding virtual crossings. See Figure 2 for the Gauss diagram of a virtual knot.

If a knot $K$ is isotopic to another knot $K^{\prime}$ then there is a sequence of moves from a diagram of $K$ to a diagram of $K^{\prime}$ as shown in Figure 3. We call these moves Reidemeister moves. We define virtual moves to be the moves of knot diagrams shown in Figure 4. A sequence of Reidemeister moves and virtual moves are called a virtual isotopy. A virtual knot is defined to be the virtual isotopy class of a virtual knot diagram.

Since a Gauss diagram defines a virtual knot diagram up to virtual moves ([4]), a virtual knot modulo virtual isotopy is equivalent to its Gauss diagram up to the moves on Gauss diagrams corresponding to the Reidemeister moves and virtual moves. 

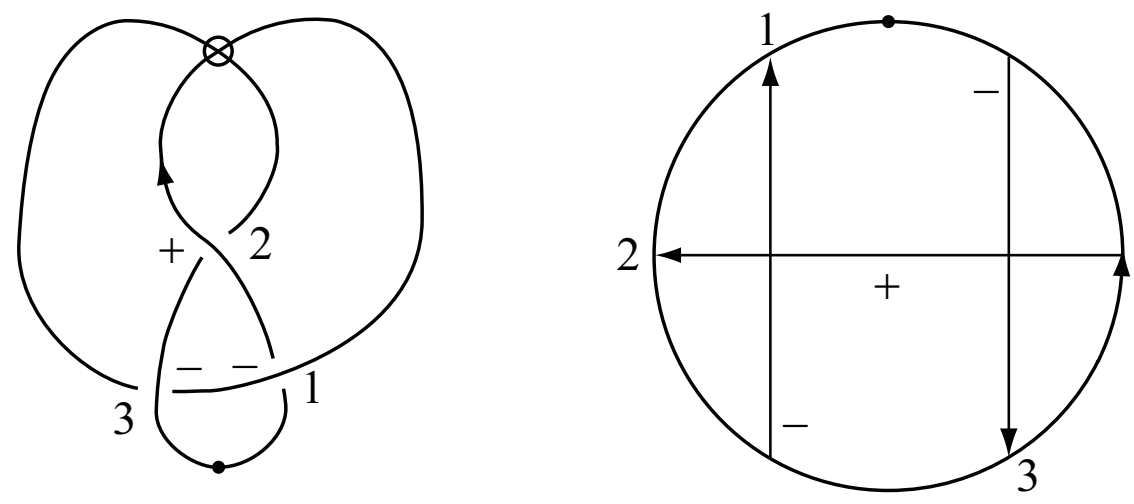

Figure 2
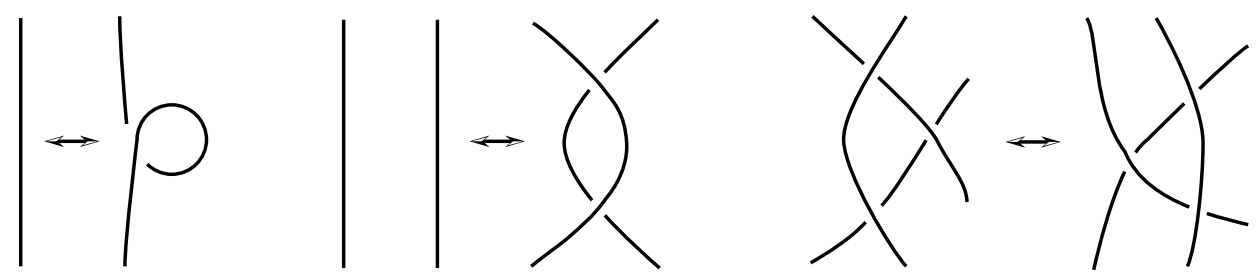

Figure 3

In [9], Kauffman showed that classical knot invariants such as fundamental groups, crystals, quandles and the bracket polynomials can be extended to virtual ones that contain all the old topological information about classical knots and are invariant under the virtual moves, giving new topological information about virtual knots and links. Kauffman ([9]) and Goussarov, Polyak and Viro ([4]) independently showed that two classical knots are isotopic if they are virtually isotopic.

A classical knot is an embedding of a circle $S^{1}$ into the 3 -dimensional Euclidean space $\mathbb{R}^{3}$. We define a long knot to be an embedding of an oriented line $\mathbb{R}$ into the Euclidean space $\mathbb{R}^{3}$. Two long knots are said to be isotopic if there is a smooth isotopy between them in the class of embeddings. A virtual long knot diagram is an immersion of the oriented line $\mathbb{R}$ into the plane whose double points are real and virtual crossings as in a virtual knot diagram. Two virtual long knot diagrams are said to be isotopic if there is a sequence of Reidemeister moves and virtual moves between them. The isotopy class of a virtual long knot diagram is called a virtual long knot. We define the Gauss diagram of a virtual long knot as that of a virtual knot, disregarding all of the virtual crossings.

As a classical link is an embedding of circles $S^{1}$ into the 3-dimensional Euclidean 

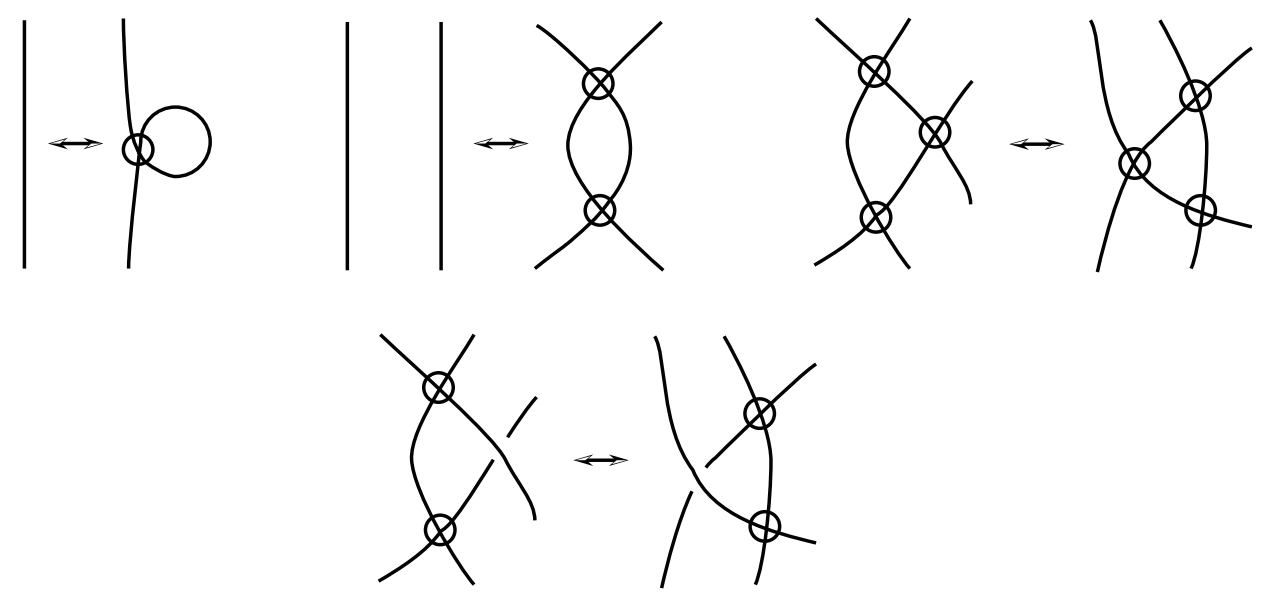

Figure 4

space $\mathbb{R}^{3}$, we can define a virtual link diagram, a virtual link and the Gauss diagram of a virtual link similarly.

In Section 2, we introduce a $t\left(a_{1}, \cdots, a_{m}\right)$-sequence of virtual knots induced from twisting of two parallel strands with some virtual crossings. Then we show that finite type invariants $v$ of virtual knots of degree $n$ have a polynomial growth of degree less than or equal to $n$. In Section 3, we study the extension of the bracket polynomial of links to virtual ones and its normalized polynomial $f_{K}(A)$ introduced by Kauffman ([9]). Then by using a $t\left(a_{1}, \cdots, a_{m}\right)$-sequence of virtual knots, we show that there are no nontrivial finite type invariants among the higher derivatives $f_{K}^{(n)}(a)$ of the $f$-polynomial $f_{K}(A)$ at any point $a$.

\section{Finite Type Invariants of Virtual Knots}

In [13], Vassiliev introduced finite type invariants of knots and in [2] Birman and Lin redefined it by using the Vassiliev skein relation. A knot or link invariant $v$ taking values in an abelian group can be extended to a singular knot or link invariant by using the Vassiliev skein relation: $v\left(K_{\times}\right)=v\left(K_{+}\right)-v\left(K_{-}\right)$, where $K_{\times}, K_{+}$and $K_{-}$are singular knot or link diagrams which are identical except the indicated local parts in Figure 5 ([1], [2]).

A knot or link invariant $v$ is called a Vassiliev invariant of degree $n$ if $n$ is the smallest nonnegative integer such that $v$ vanishes on singular knots or links with more than $n$ double points. A knot or link invariant $v$ is called a Vassiliev invariant if $v$ is a Vassiliev invariant of degree $n$ for some nonnegative integer $n$. R. Trapp showed that a Vassiliev invariant of degree $n$ has a polynomial growth of degree less 


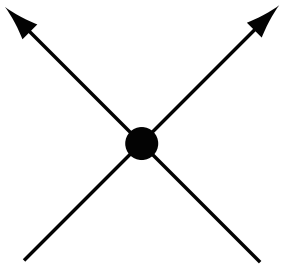

$K_{\times}$

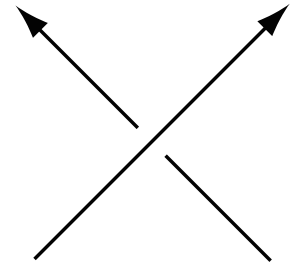

$K_{+}$

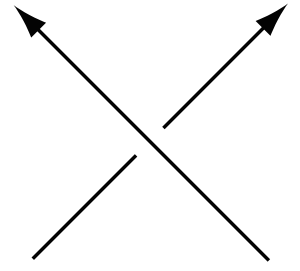

$K_{-}$

Figure 5

than or equal to $n$ on a twist sequence of knots ([12]). In [8], Kauffman introduced the bracket polynomial of links by using state models. The bracket polynomial can be extended for virtual link diagrams with the following recursive formulae $([9])$ :

(1) $<O^{n}>=\left(-A^{2}-A^{-2}\right)^{n-1}$, where $O^{n}$ is a trivial virtual knot diagram with $n$ components allowed to have some virtual crossings.

(2) $<K>=A<K_{0}>+A^{-1}<K_{\infty}>$,

where $K, K_{0}$ and $K_{\infty}$ are the virtual link diagrams identical outside of the shown parts in Figure 6.

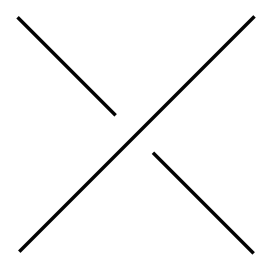

$K$
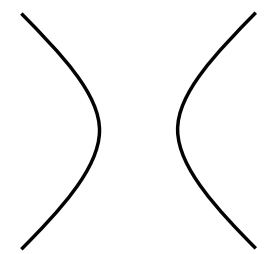

$K_{0}$

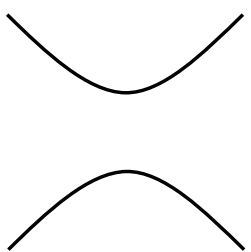

$K_{\infty}$

Figure 6

The bracket polynomials of virtual link diagrams are invariant under all but the first Reidemeister move. For an oriented virtual link diagram $K$, define its writhe $w(K)$ to be the sum of the crossing signs. The sign of a crossing in an oriented virtual knot diagram is defined as shown in Figure 7.

We define the $f$-polynomial $f_{K}(A)([9])$ of a virtual link diagram $K$ by the formula $f_{K}(A)=\left(-A^{3}\right)^{-w(K)}<K>$. Then we can easily see that the $f$-polynomial is invariant under all of the Reidemeister moves and virtual moves. For a classical link $L, V_{L}(t)=\left.f_{L}(A)\right|_{A=t^{-1 / 4}}$ is called the Jones polynomial of the link $L$. 


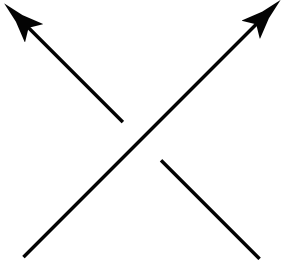

$+1$

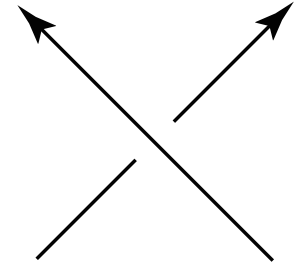

$-1$

Figure 7

There are two kinds of finite type invariants for virtual knots ([9], [4]). Kauffman introduced graphical finite type invariants as following

Definition 2.1. An invariant $v$ of rigid vertex (virtual) 4-valent graphs taking values in an abelian group is said to be a Vassiliev invariant if it satisfies that

$$
v(G \mid *)=v(G \mid+)-v(G \mid-),
$$

where $(G \mid *),(G \mid+)$ and $(G \mid-)$ are oriented graphs identical outside of the shown parts in Figure 8.

For a graph $G$ we denote the number of vertices by $N(G)$. A Vassiliev invariant is said to be of graphical finite type $n$ if $v(G)=0$ for any graph with $N(G)>n$.

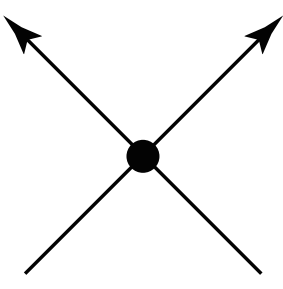

$(G \mid *)$

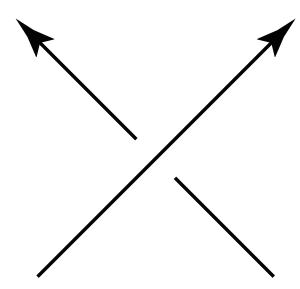

$(G \mid+)$

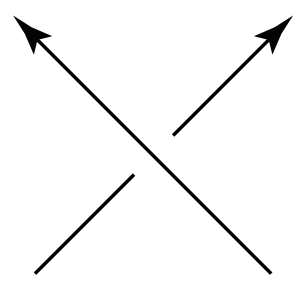

$(G \mid-)$

Figure 8

For each $n$, we can get an invariant of graphical finite type $n$ from the $f$ polynomial as following ([9]). For the quadruple $\left(K_{+}, K_{-}, K_{0}, K_{\infty}\right)$ of virtual knots which are identical except for the part shown in Figure 9, we have

$$
\left\{\begin{array}{l}
f_{K_{+}}=-A^{-2} f_{K_{0}}-A^{-4} f_{K_{\infty}}, \\
f_{K_{-}}=-A^{+2} f_{K_{0}}-A^{+4} f_{K_{\infty}} .
\end{array}\right.
$$


Therefore we see that $f_{K_{+}}\left(e^{x}\right)-f_{K_{-}}\left(e^{x}\right)$ is a multiple of $x$. Inductively we can show that the coefficient of $x^{n}$ in $f_{G}\left(e^{x}\right)$ vanishes for any rigid vertex (virtual) 4-valent graph $G$ with $N(G)>n$. Thus it is of graphical finite type $n$.

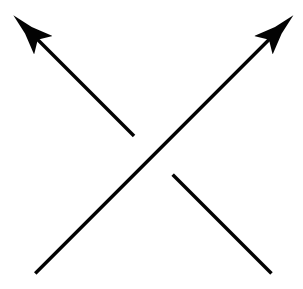

$K_{+}$

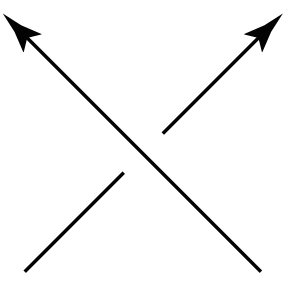

$K_{-}$
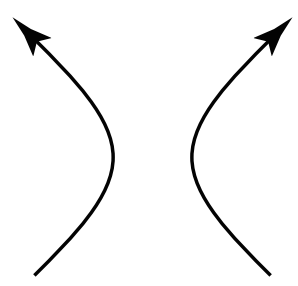

$K_{0}$

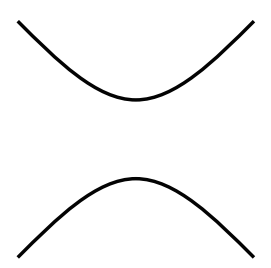

$K_{\infty}$

Figure 9

M. Goussarov, M. Polyak and O. Viro also defined finite type invariants of virtual knots by using semi-virtual crossings and gave combinatorial presentations of finite type invariants of low degrees ([4]). We denote the set of virtual knots by $\mathcal{K}$ and the set of Gauss diagrams by $\mathcal{D}$. Consider the free abelian group $\mathbb{Z} \mathcal{K}$ generated by the space $\mathcal{K}$ of virtual knots. We define a semi-virtual crossing by a formal relation as shown in Figure 10 and denote it by a real crossing surrounded by a small circle.
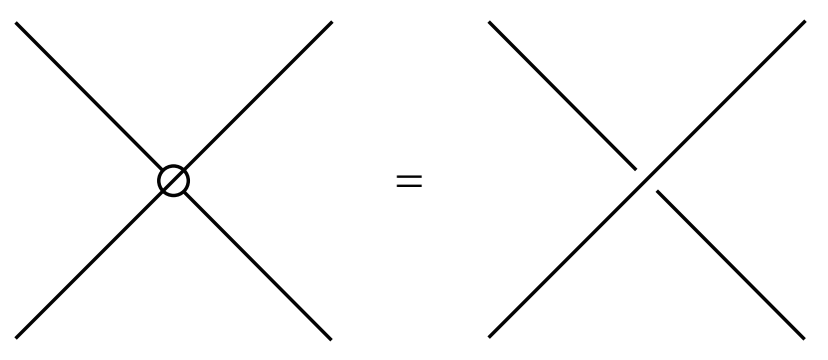

Figure 10

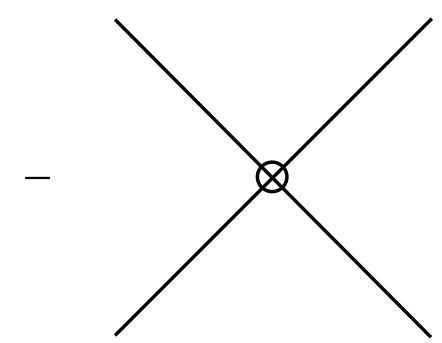

In the free abelian group generated by the set of Gauss diagrams we denote a semi-virtual crossing by a dashed arrow as in Figure 11.

Definition 2.2. Let $\mathcal{K}$ be the space of virtual knots and $\mathbb{Z} \mathcal{K}$ be the free abelian group generated by $\mathcal{K}$. For an abelian group $G$ and for a virtual knot invariant $v: \mathcal{K} \rightarrow G$, we extend the invariant $v$ to $\mathbb{Z} \mathcal{K}$ by linearity. For a natural number $n$, if $v$ vanishes for all virtual knots with more than $n$ semi-virtual crossings, then it 


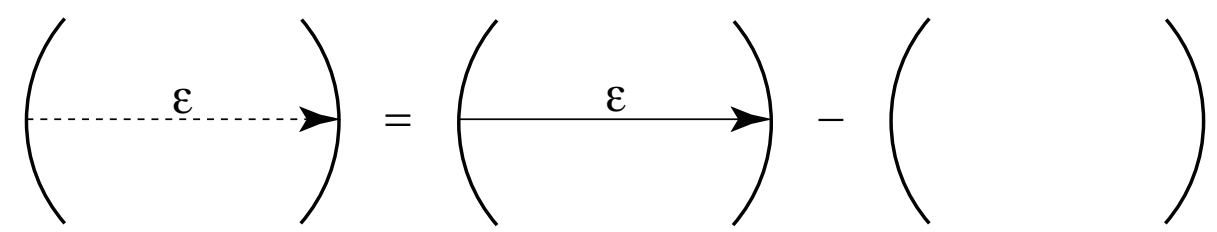

Figure 11

is called an invariant of finite type. The smallest such integer $n$ is called the degree of $v$.

Let $D$ be a virtual knot diagram and let $\left\{A_{1}, \cdots, A_{m}\right\}$ be disjoint nonempty sets of crossings of $D$ for a positive integer $m$. For $\epsilon_{k}= \pm(1 \leq k \leq m)$, let $D\left(A_{1}^{\epsilon_{1}}, \cdots, A_{m}^{\epsilon_{m}}\right)$ denote the virtual knot diagram obtained from $D$ by changing all the crossings in $A_{k}$ to virtual ones only if $\epsilon_{k}=-$. When $A$ is a singleton set $\{x\}$, we will denote $A=x$ as usual.

Let $D$ be a virtual knot diagram with crossings $x_{1}, x_{2}, \cdots, x_{n}$. Let $v$ be a finite type invariant of virtual knots of degree $<n$. By applying the skein relation for a finite type invariant of the virtual knots to the semi-virtual knot diagram obtained from $D$ by making the crossings $x_{1}, x_{2}, \cdots, x_{n}$ to the semi-virtual ones, we get the formula:

$$
\sum_{\epsilon_{i}= \pm} \epsilon_{1} \cdots \epsilon_{n} v\left(D\left(x_{1}{ }^{\epsilon_{1}}, \cdots, x_{n}{ }^{\epsilon_{n}}\right)\right)=0 .
$$

Moreover by using mathematical argument on $n$, we get the following

Lemma 2.3. Let $D$ be a virtual knot diagram with a collection $\left\{A_{1}, \cdots, A_{n}\right\}$ of disjoint nonempty sets of crossings of $D$. If $v$ is a finite type invariants of virtual knots of degree $<n$, then we have

$$
\sum_{\epsilon_{i}= \pm} \epsilon_{1} \cdots \epsilon_{n} v\left(D\left(A_{1}{ }^{\epsilon_{1}}, \cdots, A_{n}{ }^{\epsilon_{n}}\right)\right)=0 .
$$

Definition 2.4. We fix $k$ distinct points in the closed interval $[0,1]$, say $x_{1}, \cdots, x_{k}$. A virtual $(k, k)$-tangle diagram is a generic immersion of $k$ oriented curves and some oriented circles into the square $[0,1]^{2}$ with the set of endpoints of the curves to be $\left\{\left(x_{i}, y\right) \mid i=1, \cdots, k\right.$ and $\left.y=0,1\right\}$. The double points of the generic immersion are real crossings and virtual crossings. Two virtual $(k, k)$-tangle diagrams $T$ and $S$ are said to be isotopic if there is a sequence of Reidemeister moves and virtual moves from $T$ to $S$. The isotopy class of a $(k, k)$-tangle diagram is called a virtual $(k, k)$-tangle.

For two $(k, k)$-virtual tangles $S$ and $T$ with the orientations of curves of $S$ near the upper closed interval $[0,1] \times 1$ and those of the curves of $T$ near the lower closed interval $[0,1] \times 0$ are matched, we define the product $T S$ of the two tangles $T$ and $S$ 
to be the virtual tangle obtained by gluing the lower part of the square containing $S$ and the upper part of the square containing $T$.

For a virtual $(k, k)$-tangle $T$ with the orientations of the curves in the upper part of the square and those of the curves in the lower part of the square are equal, we define the closure $\bar{T}$ to be the virtual link obtained from $T$ by attaching $k$ parallel strands to the endpoints of the curves in the exterior of the square.

Definition 2.5. Let $\sigma, \sigma^{-1}$ and $\tau$ be the three virtual tangle as shown in Figure 12. For nonzero integers $a_{1}, \cdots, a_{m}$ we define the virtual tangle $T\left(a_{1}, \cdots, a_{m}\right)$ by

$T\left(a_{1}, \cdots, a_{m}\right)=\left\{\begin{aligned} & \sigma^{a_{1}} \tau \sigma^{a_{2}} \tau \cdots \tau \sigma^{a_{m}} \text { if } \overline{\sigma^{a_{1}} \tau \sigma^{a_{2}} \tau \cdots \tau \sigma^{a_{m}}} \text { is a two components } \\ & \text { virtual link, } \\ & \sigma^{a_{1}} \tau \sigma^{a_{2}} \tau \cdots \tau \sigma^{a_{m}} \tau \text { if } \overline{\sigma^{a_{1}} \tau \sigma^{a_{2}} \tau \cdots \tau \sigma^{a_{m}}} \text { is a virtual knot. }\end{aligned}\right.$

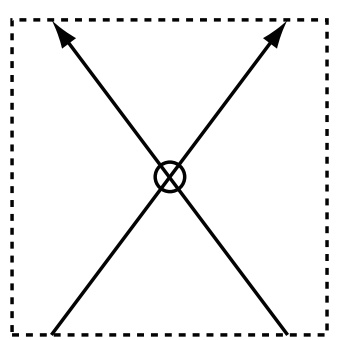

$\tau$

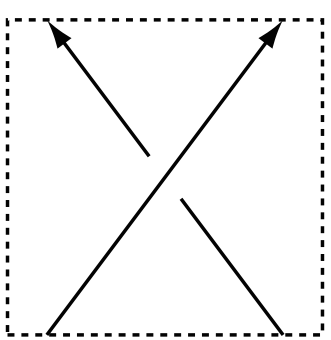

$\sigma$

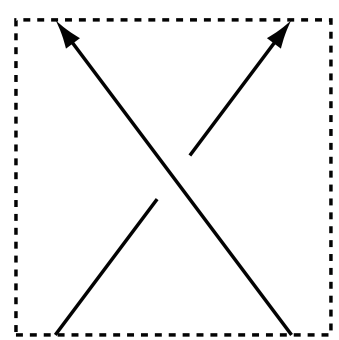

$\sigma^{-1}$

Figure 12

Definition 2.6. A sequence $\left\{K_{i}\right\}_{i=0}^{\infty}$ of virtual knots is called a $t\left(a_{1}, \cdots, a_{m}\right)$ sequence if there is a tangle $S$ such that $K_{i}=\overline{S T^{i}}$, where $T=T\left(a_{1}, \cdots, a_{m}\right)$ and $T^{i}=T T \cdots T$ is the $i$-times self-product of $T$ for $i \in \mathbb{N}$ and $T^{0}$ denotes the trivial tangle with the orientation inherited from $T$.

Throughout this section, unless otherwise stated, we fix an $m$-tuple $\left(a_{1}, a_{2}, \cdots, a_{m}\right)$ of nonzero integers and fix a $t\left(a_{1}, \cdots, a_{m}\right)$-sequence $\left\{K_{i}\right\}_{i=0}^{\infty}$ such that $K_{i}=\overline{S T^{i}}$ for some tangle $S$, where $T$ is the tangle $T\left(a_{1}, \cdots, a_{m}\right)$.

Let $A$ be the set of crossings of $T$. For each fixed $K_{i}$, let $T_{j}(0<j \leq i)$ be the $j$-th $T$ from the bottom of the diagram of $T^{i}$ and let $A_{j}$ be the set of crossings in $T_{j}$ corresponding to $A$ in $T$.

For each $j \in \mathbb{N}$, define $\Delta^{j} K_{i}$ to be the semi-virtual knot obtained from $K_{j+i}$ by changing each crossing in $\bigcup_{l=1}^{j} A_{l}$ to a semi-virtual one. Let $v$ be an invariant of 
virtual knots. Define $\bar{v}$ on $\{$ virtual knots $\} \bigcup\left\{\Delta^{j} K_{i} \mid i, j \in \mathbb{N}\right\}$ by setting

$$
\begin{aligned}
& \left.\bar{v}\right|_{\{\text {virtual knots }\}}=v, \\
& \bar{v}\left(\Delta^{0} K_{i}\right)=v\left(K_{i}\right) \text { and } \\
& \bar{v}\left(\Delta^{j} K_{i}\right)=\bar{v}\left(\Delta^{j-1} K_{i+1}\right)-\bar{v}\left(\Delta^{j-1} K_{i}\right) \text { inductively. }
\end{aligned}
$$

Definition 2.7. Let $\left\{a_{i}\right\}_{i=0}^{\infty}$ be a sequence of numbers, define $\Delta^{0} a_{i}=a_{i}$ and $\Delta^{n+1} a_{i}=\Delta^{n} a_{i+1}-\Delta^{n} a_{i}$ inductively for $n \in \mathbb{N}$. The sequence $\left\{\Delta^{n} a_{i}\right\}_{i=0}^{\infty}$ is called the $n$-th difference sequence of $\left\{a_{i}\right\}_{i=0}^{\infty}$. A sequence $\left\{a_{i}\right\}_{i=0}^{\infty}$ of numbers is said to have a polynomial growth if there exist a polynomial $f(x)$ such that $a_{i}=f(i)$ for all $i \in \mathbb{N}$.

The Newton's formula says that $a_{i}=\sum_{j=0}^{i}\left(\begin{array}{l}i \\ j\end{array}\right) \Delta^{j} a_{0}$ ([3]) so the highest order term of $i$ in the summation is $\left(\begin{array}{l}i \\ n\end{array}\right) \Delta^{n} a_{0}$ if $\left\{\Delta^{n+1} a_{i}\right\}$ vanishes. Moreover we see that $\Delta^{n+1} a_{i}=0$ for all $i$ if and only if $a_{i}$ is a polynomial in $i$ of degree $\leq n$.

Lemma 2.8. Let $v$ be a virtual knot invariant and let $\left\{K_{i}\right\}_{i=0}^{\infty}$ be a $t\left(a_{1}, \cdots, a_{m}\right)$ sequence of virtual knots. Let $T$ be the tangle $T\left(a_{1}, \cdots, a_{m}\right)$. Let $S$ be a tangle such that $K_{i}=\overline{S T^{i}}$ for each $i$. Define $\Delta^{j} K_{i}$ and $\bar{v}$ as above. Then for each $j \in \mathbb{N}$, $\bar{v}\left(\Delta^{j}\left(K_{i}\right)\right)=\Delta^{j}\left(\bar{v}\left(K_{i}\right)\right)$.

Proof. We will use the induction argument on $j$. If $j=0, \bar{v}\left(\Delta^{0}\left(K_{i}\right)\right)=v\left(K_{i}\right)=$ $\Delta^{0}\left(\bar{v}\left(K_{i}\right)\right)$. Assume that it holds for $j=1, \cdots, n-1$. Then $\bar{v}\left(\Delta^{n}\left(K_{i}\right)\right)=$ $\bar{v}\left(\Delta^{n-1}\left(K_{i+1}\right)\right)-\bar{v}\left(\Delta^{n-1}\left(K_{i}\right)\right)=\Delta^{n-1}\left(\bar{v}\left(K_{i+1}\right)\right)-\Delta^{n-1}\left(\bar{v}\left(K_{i}\right)\right)$

$=\Delta^{n}\left(\bar{v}\left(K_{i}\right)\right)$.

Now we will evaluate $\bar{v}\left(\Delta^{n}\left(K_{i}\right)\right)$ by resolving the sets of semi-virtual crossings. For each $i \in \mathbb{N}, K_{i}=K_{i}\left(A_{1}^{+}, A_{2}, \cdots, A_{i}\right)=K_{i+1}\left(A_{1}^{-}, A_{2}, \cdots, A_{i+1}\right)$. Then we have

$$
\begin{aligned}
\bar{v}\left(\Delta^{1}\left(K_{i}\right)\right) & =\bar{v}\left(\Delta^{0}\left(K_{i+1}\right)\right)-\bar{v}\left(\Delta^{0}\left(K_{i}\right)\right) \\
& =v\left(K_{i+1}\right)-v\left(K_{i}\right) \\
& =\bar{v}\left(K_{i+1}\left(A_{1}^{+}, A_{2}, \cdots, A_{i+1}\right)\right)-\bar{v}\left(K_{i+1}\left(A_{1}^{-}, A_{2}, \cdots, A_{i+1}\right)\right) .
\end{aligned}
$$

Now we assume that for $1 \leq j \leq n$ the following holds;

$$
\bar{v}\left(\Delta^{j}\left(K_{i}\right)\right)=\sum_{\epsilon_{i}= \pm} \epsilon_{1} \cdots \epsilon_{j} \bar{v}\left(K_{j+i}\left(A_{1} \epsilon_{1}, \cdots, A_{j}{ }^{\epsilon_{j}}, A_{j+1}, \cdots, A_{j+i}\right)\right) .
$$

Then we see that

$$
\begin{aligned}
& \bar{v}\left(\Delta^{n+1}\left(K_{i}\right)\right) \\
= & \bar{v}\left(\Delta^{n}\left(K_{i+1}\right)\right)-\bar{v}\left(\Delta^{n}\left(K_{i}\right)\right) \\
= & \sum_{\epsilon_{i}= \pm} \epsilon_{1} \cdots \epsilon_{n} \bar{v}\left(K_{n+i+1}\left(A_{1}{ }_{1}, \cdots, A_{n}{ }^{\epsilon_{n}}, A_{n+1}, \cdots, A_{n+i+1}\right)\right) \\
& -\sum_{\epsilon_{i}= \pm} \epsilon_{1} \cdots \epsilon_{n} \bar{v}\left(K_{n+i}\left(A_{1}{ }^{\epsilon_{1}}, \cdots, A_{n}{ }^{\epsilon_{n}}, A_{n+1}, \cdots, A_{n+i}\right)\right)
\end{aligned}
$$




$$
\begin{aligned}
= & \sum_{\epsilon_{i}= \pm} \epsilon_{1} \cdots \epsilon_{n} \bar{v}\left(K_{n+i+1}\left(A_{1}{ }^{\epsilon_{1}}, \cdots, A_{n}{ }^{\epsilon_{n}}, A_{n+1}^{+}, A_{n+2}, \cdots, A_{n+i+1}\right)\right) \\
& -\sum_{\epsilon_{i}= \pm} \epsilon_{1} \cdots \epsilon_{n} \bar{v}\left(K_{n+i+1}\left(A_{1}{ }^{\epsilon_{1}}, \cdots, A_{n}^{\epsilon_{n}}, A_{n+1}^{-}, A_{n+2}, \cdots, A_{n+i+1}\right)\right) \\
= & \sum_{\epsilon_{i}= \pm} \epsilon_{1} \cdots \epsilon_{n+1} \bar{v}\left(K_{n+i+1}\left(A_{1} \epsilon_{1}, \cdots, A_{n}{ }^{\epsilon_{n}}, A_{n+1}^{\epsilon_{n+1}}, A_{n+2}, \cdots, A_{n+i+1}\right)\right) .
\end{aligned}
$$

We have proved the following

Lemma 2.9. Let $v$ be a virtual knot invariant and let $\left\{K_{i}\right\}_{i=0}^{\infty}$ be a $t\left(a_{1}, \cdots, a_{m}\right)$ sequence of virtual knots. Let $T$ be the tangle $T\left(a_{1}, \cdots, a_{m}\right)$ and let $S$ be a tangle such that $K_{i}=\overline{S T^{i}}$ for each $i$. Then for $i=0,1, \cdots$ and for $n=1,2, \cdots$, we get

$$
\bar{v}\left(\Delta^{n}\left(K_{i}\right)\right)=\sum_{\epsilon_{i}= \pm} \epsilon_{1} \cdots \epsilon_{n} \bar{v}\left(K_{n+i}\left(A_{1}{ }^{\epsilon_{1}}, \cdots, A_{n}{ }^{\epsilon_{n}}, A_{n+1}, \cdots, A_{n+i}\right)\right) .
$$

Theorem 2.10. Let $\left\{K_{i}\right\}_{i=0}^{\infty}$ be a $t\left(a_{1}, \cdots, a_{m}\right)$-sequence of virtual knots for any nonzero integers $a_{1}, \cdots, a_{m}$. Then any finite type invariant $v$ of virtual knots of degree $n$ has a polynomial growth on $\left\{K_{i}\right\}_{i=0}^{\infty}$ of degree $\leq n$.

Proof. By the Newton's formula, it is sufficient to show that $\Delta^{n+1} v\left(K_{i}\right)=0$, which comes from Lemmas 2.3, 2.8 and 2.9:

$$
\begin{aligned}
\Delta^{n+1} v\left(K_{i}\right) & =\Delta^{n+1} \bar{v}\left(K_{i}\right) \\
& =\bar{v}\left(\Delta^{n+1}\left(K_{i}\right)\right) \\
& =\sum_{\epsilon_{i}= \pm} \epsilon_{1} \cdots \epsilon_{n+1} \bar{v}\left(K_{n+i+1}\left(A_{1} \epsilon_{1}, \cdots, A_{n+1}^{\epsilon_{n+1}}, A_{n+2}, \cdots, A_{n+i+1}\right)\right) \\
& =\sum_{\epsilon_{i}= \pm} \epsilon_{1} \cdots \epsilon_{n+1} v\left(K_{n+i+1}\left(A_{1}{ }^{\epsilon_{1}}, \cdots, A_{n+1}^{\epsilon_{n+1}}, A_{n+2}, \cdots, A_{n+i+1}\right)\right) \\
& =0 .
\end{aligned}
$$

Theorem 2.10 holds for virtual long knots, when we define finite type invariants and $t\left(a_{1}, \cdots, a_{m}\right)$-sequence of virtual long knots in the same way.

\section{The $f$-polynomials and Finite Type Invariants of Virtual Knots}

In [2], Birman and Lin showed that the coefficient of $x^{n}$ in the Maclaurin series of the polynomial $V_{K}\left(e^{x}\right)$ in $e^{x}$ is a Vassiliev invariant of type less than or equal to $n$, where $V_{K}(t)$ is the Jones polynomial of a knot $K([5])$. Therefore we see 
that the $n$-th derivative $V_{K}^{(n)}(1)$ of the Jones polynomial $V_{K}(t)$ of a knot $K$ at 1 is a Vassiliev invariant of degree less than or equal to $n$. Park and the first author showed that the $n$-th derivative $V_{K}^{(n)}(a)$ of the Jones polynomial at $t=a$ is not a Vassiliev invariant for $a \neq 1([7])$. The Jones polynomial was extended to the $f$-polynomial of virtual knots by Kauffman $([9])$.

For each $n$, the coefficient of $x^{n}$ of $f_{K}\left(e^{x}\right)$ is of graphical finite type $n$ and so $f_{K}^{(n)}(1)$ is of graphical finite type $n$. In this section we show that the $n$-th derivative $f_{K}^{(n)}(1)$ of the $f$-polynomial $f_{K}(A)$ of a knot at $A=1$ is not an invariant of a finite type for $n \geq 2$ by using a $t(1)$-sequence of virtual knots, following the definition of finite type invariants introduced by Goussarov, Polyak and Viro.

By using the recursive formula for the $f$-polynomial to evaluate $f_{K}^{(n)}(1)$, we see that $f_{K}(1)=1$ and $f_{K}^{\prime}(1)=0$ for all knots $K$. See Theorem 13 in [9] and refer [10] for more details.

Let $S$ and $T$ be the tangle diagram as shown in Figure 13 . Let $K_{i}$ be the diagram of a virtual knot $\overline{S T^{i}}$ obtained by closing the product $S T^{i}$ of $S$ and $T^{i}$, where $T^{i}$ is the $i$-times self-product of the tangle $T$ for $i \geq 0$. In particular $K_{0}$ and $K_{1}$ are trivial virtual knot diagrams.

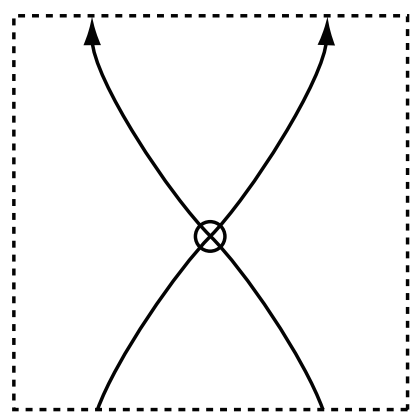

$S$

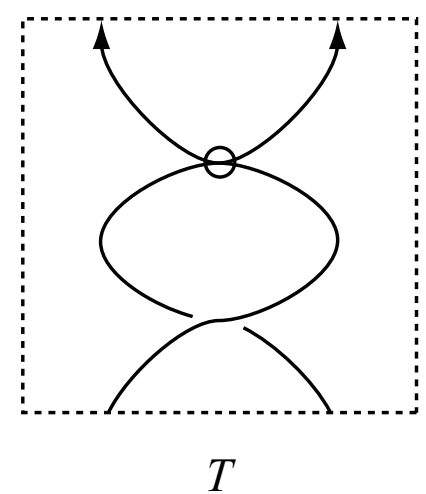

$T$

Figure 13

Lemma 3.1. We have the following recursive formula of the $f$-polynomials for the sequence $\left\{K_{i}\right\}_{i=0}^{\infty}$ of virtual knots.

$$
\left\{\begin{array}{l}
f_{K_{0}}(A)=1=f_{K_{1}}(A) \\
f_{K_{i+1}}(A)=A^{-4} f_{K_{i-1}}(A)-A^{-6 i-4}+A^{-6 i}
\end{array}\right.
$$

Proof. For each natural number $i$, let $U_{i}$ be the virtual knot diagram obtained from the tangle $T^{i}$ as shown in Figure 14. 
Then we can easily see that $\left\langle U_{i}\right\rangle=\left(-A^{3}\right)^{-i}$. By applying the recursive formula of the bracket polynomials to a crossing of the diagram $K_{i+1}=\overline{S T^{i+1}}$, we have

$$
\begin{aligned}
<K_{i+1}> & =A<\overline{T^{i}}>+A^{-1}<U_{i}> \\
& =A^{2}<\overline{S T^{i-1}}>+<U_{i-1}>+A^{-1}<U_{i}> \\
& =A^{2}<K_{i-1}>+\left(-A^{3}\right)^{-i+1}+A^{-1}\left(-A^{3}\right)^{-i}
\end{aligned}
$$

Since $f_{K_{i}}(A)=\left(-A^{3}\right)^{-i}<K_{i}>$ for each $i$, we have $f_{K_{i+1}}(A)=A^{-4} f_{K_{i-1}}(A)-$ $A^{-6 i-4}+A^{-6 i}$.

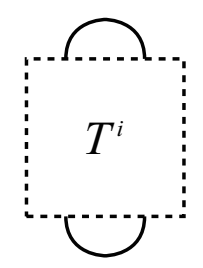

$U_{i}$

Figure 14

We find a recursive formula for the derivative $f_{K_{i}}^{(n)}(1)$ of the $f$-polynomial for the sequence $\left\{K_{i}\right\}_{i=0}^{\infty}$ of virtual knots.

Lemma 3.2. With notations as above, for each natural number $j$ we have

$$
\left\{\begin{array}{l}
f_{K_{2 j}}(A)=A^{-4 j}-\left(A^{-12 j}-A^{-4 j}\right)\left(A^{2}+A^{-2}\right)^{-1} \text { and } \\
f_{K_{2 j+1}}(A)=A^{-4 j}-\left(A^{-12 j-6}-A^{-4 j-6}\right)\left(A^{2}+A^{-2}\right)^{-1} .
\end{array}\right.
$$

Proof. From Lemma 3.1, we see that

$$
A^{2(i+1)} f_{K_{i+1}}(A)=A^{2(i-1)} f_{K_{i-1}}(A)-A^{-4 i-2}+A^{-4 i+2} .
$$

Put $b_{i}=A^{2 i} f_{K_{i}}(A)$ for each $i \in \mathbb{N}$. Then we have $b_{i+1}-b_{i-1}=-A^{-4 i-2}+A^{-4 i+2}$, $b_{0}=1$ and $b_{1}=A^{2}$.

For an even number $i=2 j$, we have $b_{2 j+1}-b_{2 j-1}=-A^{-8 j-2}+A^{-8 j+2}$. Then

$$
\begin{aligned}
b_{2 j+1} & =b_{1}+\left(b_{3}-b_{1}\right)+\cdots+\left(b_{2 j+1}-b_{2 j-1}\right) \\
& =A^{2}+\sum_{k=1}^{j}\left(-A^{-8 k-2}+A^{-8 k+2}\right) \\
& =A^{2}+\frac{\left(1-A^{-8 j}\right)}{A^{6}+A^{2}}
\end{aligned}
$$


Therefore we have $f_{K_{2 j+1}}=\frac{1}{A^{2(2 j+1)}} b_{2 j+1}=A^{-4 j}-\left(A^{-12 j-6}-A^{-4 j-6}\right)\left(A^{2}+\right.$ $\left.A^{-2}\right)^{-1}$.

For an odd natural number $i=2 j+1$, we get $b_{2 j+2}-b_{2 j}=-A^{-8 j-6}+A^{-8 j-2}=$ $A^{-4}\left(b_{2 j+1}-b_{2 j-1}\right)$. Therefore we see that $b_{2 j}=1+\frac{1-A^{-8 j}}{A^{2}+A^{-2}}$ and $f_{K_{2} j}=A^{-4 j}-$ $\left(A^{-12 j}-A^{-4 j}\right)\left(A^{2}+A^{-2}\right)^{-1}$.

By evaluating the growth of the $n$-th derivative $f_{K}^{(n)}(1)$ of the $f$-polynomial at $A=1$ on the $t(1)$-sequence $\left\{K_{i}\right\}_{i=0}^{\infty}$ we get the following

Theorem 3.3. The $n$-th derivative $f_{K}^{(n)}(1)$ of the $f$-polynomial of a knot $K$ at $A=1$ is not of finite type for $n \geq 2$.

Proof. Let $n$ be a fixed integer greater than or equal to 2. From Theorem 2.10, we see that it is sufficient to show that $f_{K}^{(n)}(1)$ does not have a polynomial growth on the sequence $\left\{K_{i}\right\}_{i=0}^{\infty}$. Actually, we see that $f_{K}^{(n)}(1)$ has polynomial growths on the two sequences $\left\{K_{2 j}\right\}_{j=0}^{\infty}$ and $\left\{K_{2 j+1}\right\}_{j=0}^{\infty}$ from Lemma 3.2. However we show that it does not have a polynomial growth on $\left\{K_{i}\right\}_{i=0}^{\infty}$. For an integer $k$ and a positive integer $l$, we denote the number $k(k-1) \cdots(k-l+1)$ by $P(k, l)$ and we also use the convention $P(k, 0)=1$. Suppose that the derivative $f_{K}^{(n)}(1)$ have a polynomial growth on $\left\{K_{i}\right\}_{i=0}^{\infty}$. Then by comparing the $n$-th derivative of the formula in Lemma 3.2 at $A=1$, we get the equation:

$$
\begin{gathered}
P(-4 j, n)-P(-4 j+2, n) \\
+\left.\sum_{r=0}^{n}\left(\begin{array}{l}
n \\
r
\end{array}\right)(P(-4 j, r)-P(-4 j-4, r))\left\{\left(A^{2}+A^{-2}\right)^{(-1)}\right\}^{(n-r)}\right|_{A=1}=0 .
\end{gathered}
$$

However the coefficient of $j^{n-2}$ in the left hand side of the above equation is $(-4)^{n-2}\left(-\frac{1}{4}\right)(3 A-2 B-C)=(-4)^{n-3}\left(-24 n^{2}+24 n\right) \neq 0$, where $A, B$, and $C$ are polynomials in $n$ given as follows;

$$
\begin{aligned}
A & =\left(\sum_{k=0}^{n-1}(-k)\right)^{2}-\sum_{k=0}^{n-1}(-k)^{2}, \\
B & =\left(\sum_{k=-2}^{n-3}(-k)\right)^{2}-\sum_{k=-2}^{n-3}(-k)^{2} \text { and } \\
C & =\left(\sum_{k=4}^{n+3}(-k)\right)^{2}-\sum_{k=4}^{n+3}(-k)^{2} .
\end{aligned}
$$

Since the $n$-th derivative $f_{K}^{(n)}(1)$ of the $f$-polynomial at $A=1$ on the $t(1)$-sequence $\left\{K_{i}\right\}_{i=0}^{\infty}$ does not have a polynomial growth, by Theorem $2.10, f_{K}^{(n)}(1)$ is not a finite type invariant of virtual knots. 
Since the restriction of a finite type invariant of virtual knots to the set of knots is a Vassiliev invariant ([4]) and since the derivative $V_{K}^{(n)}(a)$ of the Jones polynomial $V_{K}(t)$ at $t=a$ is not a Vassiliev invariant for $a \neq 1([7]), f_{K}^{(n)}(a)$ is not of finite type for $a \neq 1$. Refer [4], [14], [6] and [7] for more details. Hence we get the following

Corollary 3.4. Let $f_{K}(A)$ be the $f$-polynomial of a virtual knot $K$. For a natural number $n$, the $n$-th derivative $f_{K}^{(n)}(a)$ of the $f$-polynomial of a virtual knot $K$ at a point $a$ is of finite type if and only if $n \leq 1$ and $a=1$.

Acknowledgements. The first author was supported by the Ministry of Science, ICT and Future Planning.

\section{References}

[1] D. Bar-Natan, On the Vassiliev knot invariant, Topology, 34 (1995), 423-427.

[2] J. S. Birman and X.-S. Lin, Knot polynomials and Vassiliev's invariants, Invent. Math., 111(1993), 225-270.

[3] L. Brand, Differential and Difference equations, John Wiley \& Sons, Inc., New York, London, Sydney, 1966.

[4] M. Goussarov, M. Polyak and O. Viro, Finite type invariants of classical and virtual knots, Topology, 39(2000), 1045-1068.

[5] V. F. R. Jones, A polynomial invariant for knots via von Neumann algebras, Bull. Amer. Math. Soc., 12(1985), 103-112.

[6] M.--J. Jeong and C.-Y. Park, Vassiliev invariants and double dating tangles, J. of Knot Theory and Its Ramifications, 11(4)(2002), 527-544.

[7] M.--J. Jeong and C.-Y. Park, Vassiliev invariants and Knot polynomials, Topology and Its Applications, 124(3)(2002), 505-521.

[8] L. H. Kauffman, State models and the Jones polynomial, Topology, 26(1987), 395407.

[9] L. H. Kauffman, Virtual knot theory, Europ. J. Combinatorics, 20(1999), 663-691.

[10] H. Murakami, On derivatives of the Jones polynomial, Kobe J. Math., 3(1986), 61-64.

[11] K. Murasugi, Knot Theory and Its Applications, Birkhäuser, 1996.

[12] R. Trapp, Twist sequences and Vassiliev invariants, J. Knot Theory and Its Ramif., 3(1994), 391-405.

[13] V. A. Vassiliev, Cohomology of knot spaces, Theory of Singularities and It's Applications, edited by V. I. Arnold, Advances in Soviet Mathematics, Vol. 1, AMS, 1990.

[14] J. Zhu, On Jones knot invariants and Vassiliev invariants, New Zealand J. Math., 27(1998), 294-299. 